\title{
DETERMINAÇÃO EXPERIMENTAL DA SOLUBILIDADE DE $\alpha$-TOCOFEROL EM MISTURAS DE ETANOL+ÁGUA
}

\author{
I. H. N. MORAIS ${ }^{1}$ e R. A. MALAGONI ${ }^{1}$ \\ ${ }^{1}$ Universidade Federal de Uberlândia, Faculdade de Engenharia Química \\ E-mail para contato: malagoni@ufu.br
}

\begin{abstract}
RESUMO - O composto $\alpha$-Tocoferol, popularmente conhecido como vitamina E, é um composto lipossolúvel, ou seja, solubiliza-se em lipídeos. O grande interesse por esta vitamina se deve ao fato das inúmeras funções que ela desempenha no organismo humano, devido ao seu forte potencial antioxidante ela atua na diminuição do processo de envelhecimento das células e na proteção de doenças crônicas não transmissíveis como o câncer, doenças cardiovasculares e o mal de Parkinson. Por se tratar de uma molécula apolar, a sua solubilidade em água é muito baixa e pode se tornar maior quando um solvente apolar é colocado juntamente a água. Este trabalho, ainda em andamento, visa estudar a solubilidade da vitamina $\mathrm{E}$ em misturas etanol+água com frações de 25 e $50 \%$ de etanol, em temperaturas de 20 a $40^{\circ} \mathrm{C}$, inclusive na temperatura do corpo humano $36,7^{\circ} \mathrm{C}$. Com os resultados obtidos foi possível concluir que a solubilidade da vitamina $\mathrm{E}$ aumenta consideravelmente com o aumento da concentração de etanol na solução, mas estudos e experimentos ainda são necessários para verificar se há uma relação entre o aumento da temperatura com o aumento da solubilidade.
\end{abstract}

\section{INTRODUÇÃO}

O termo vitamina E descreve uma família de oito antioxidantes: quatro tocoferóis (alfa, beta, gama e delta) e quatro tocotrienóis (alfa, beta, gama e delta). O alfa-tocoferol é a única forma de vitamina $\mathrm{E}$ produzida pelo corpo humano, logo, é a forma de vitamina E encontrada em maiores quantidades no sangue (Traber, 1999).

A vitamina E ou $\alpha$-Tocoferol é um composto lipossolúvel, ou seja, solubiliza-se em lipídeos. Sua molécula é constituída por uma cadeia grande de alcano com um grupo éter e um grupo fenol. Embora esses grupos possam formar ligações de hidrogênio com a água, a cadeia de alcano apresenta tamanho considerável para que essa molécula atue como um composto hidrofóbico (apolar) reduzindo a solubilidade da mesma em água. Porém, quando se adiciona outro solvente junto à água, como, por exemplo, o etanol, ocorre um grande aumento da solubilidade, pois surgem interações apolares entre o etanol e a mólecula de vitamina.

Estudos mostram que para uma fração mássica de etanol de $10 \%$, a solubilidade aumenta 1,5 vezes e maiores efeitos podem ser observados em altas concentrações de etanol, por exemplo, para uma fração de etanol de $70 \%$, a solubilidade aumenta aproximadamente 450 vezes devido à atração entre as cadeias de alcanos da vitamina e as moléculas de etanol 
(Gupta e Heidemann, 1990). A Tabela 1 apresenta a estrutura e a massa molecular dessa vitamina.

Tabela 1 - Fórmula estrutural e massa molecular da vitamina E

Nome

O estudo da solubilidade da vitamina E pode ser feito com o uso de células de equilíbrio semelhantes às utilizadas em Malagoni (2006) e Oliveira et al. (2013). O método utilizado nesses trabalhos proporcionou a obtenção de dados de solubilidade confiáveis tanto para solutos orgânicos e inorgânicos.

O objetivo deste trabalho foi determinar experimentalmente a solubilidade da vitamina E em misturas de etanol+água, frações de etanol de 25 e $50 \%$, utilizando uma célula de equilíbrio que operou em temperaturas de 20 a $40^{\circ} \mathrm{C}$, inclusive na temperatura do corpo humano: $36,7^{\circ} \mathrm{C}$. Para isto, fez-se necessário a investigação das variáveis tempo de agitação e de repouso da solução no interior da célula de equilíbrio.

\section{MATERIAL E MÉTODOS}

Primeiramente foi realizada uma pesquisa na literatura sobre qual o comprimento de onda seria utilizado. Foram encontrados dois resultados satisfatórios para se trabalhar com a vitamina E, 291,6 e $300 \mathrm{~nm}$. Optou-se por trabalhar com 291,6 $\mathrm{nm}$ por se um comprimento de onda já relatado por Dubbs e Gupta (1998). Logo após foram realizados estudos acerca da solubilidade da vitamina E em misturas etanol+água com frações de etanol de 25 e $50 \%$, visando investigar o aumento da solubilidade com o aumento das frações de etanol. Para isto, as variáveis tempo de agitação e de equilíbrio também foram investigadas.

Após o estudo das variáveis tempo de agitação e de equilíbrio adotou-se os valores de $24 \mathrm{~h}$ de agitação e $6 \mathrm{~h}$ de repouso, para a realização dos experimentos, com o intuito de englobar a faixa de tempo utilizada por diversos autores. Nos experimentos de Oliveira et al (2013), o tempo de agitação e de repouso utilizados foram $24 \mathrm{~h}$ e $2 \mathrm{~h}$ respectivamente, estes tempos apresentaram resultados constantes de solubilidade, o que comprovou a saturação do meio, observa-se que o tempo de repouso foi aumentado para verificar a sua influência na solubilidade.

Os ensaios de solubilidade da vitamina E em soluções com 25 e $50 \%$ de etanol foram realizados em uma célula de equilibro encamisada de vidro borossilicato e com capacidade de $40 \mathrm{~mL}$. Para isso, a célula operou em diferentes temperaturas, 20 a $40^{\circ} \mathrm{C}$. Um banho termostatizado (TECNAL, TE-184), manteve a temperatura do sistema constante. Um agitador magnético (TECNAL, TE-0851) e uma barra magnética revestida com teflon promoveram a homogeneização da mistura durante o experimento. Para cada temperatura estudada, três amostras de solução saturada de vitamina foram coletadas, medindo-se na 
sequência as respectivas absorbâncias no espectrofotômetro (T60, UV-Spectrophotometer-PG Instruments).

A Tabela 2 apresenta os reagentes e seus fabricantes utilizados nos experimentos.

Tabela 2 - Reagentes utilizados no estudo de solubilidade da vitamina E

\begin{tabular}{ll}
\hline Substância/Fabricante & Pureza \\
\hline Etanol/Vetec & $95,0 \%$ \\
Vitamina E/Sigma & $99,9 \%$ \\
Água bi-destilada e deionizada & - \\
\hline
\end{tabular}

$\mathrm{Na}$ célula, inicialmente, acrescentava-se em excesso a vitamina E e depois a solução etanol+água. Um termopar previamente calibrado e acoplado a um indicador de temperatura (Full Gauge Controls, TIC 17RGTi) foi usado para se garantir a temperatura da solução. O agitador foi ligado quando se atingiu a temperatura desejada. A unidade experimental utilizada neste trabalho está representada na Figura 1.

Figura 1 - Unidade experimental de solubilidade.

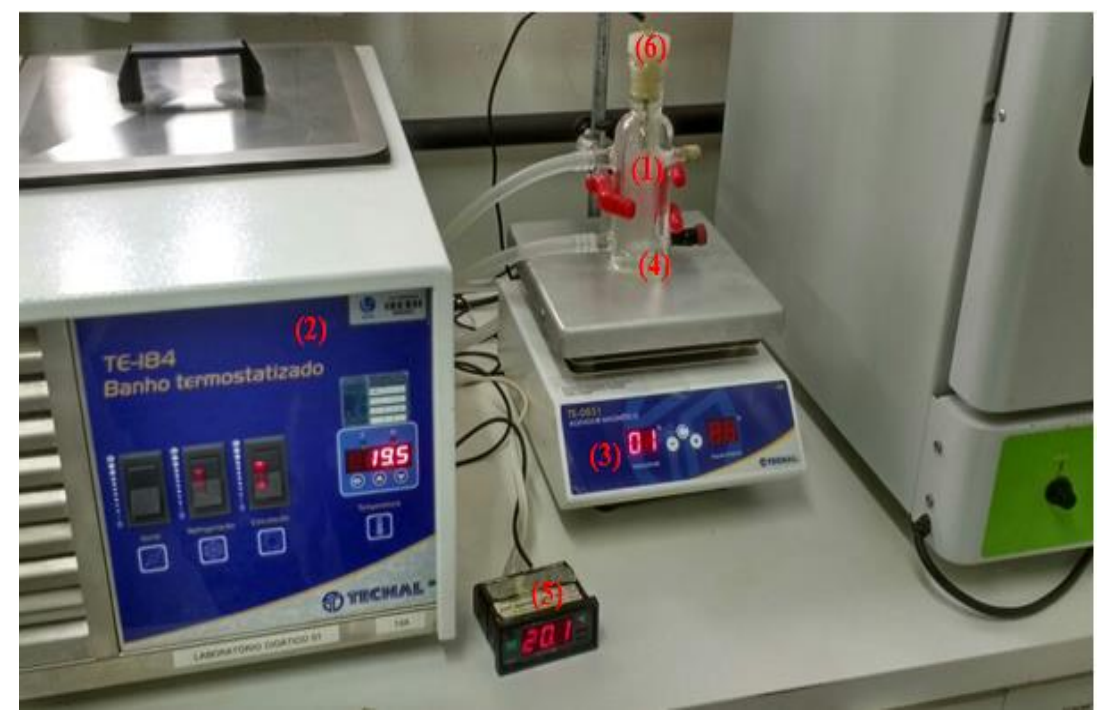

Na Figura 1, observa-se que (1) é a célula de equilíbrio, (2) é o banho termostatizado, (3) o agitador magnético, (4) barra magnética, (5) indicador de temperatura e (6) rolha de tecnyl.

\section{RESULTADOS E DISCUSSÃO}

A Tabela 3 apresenta os valores de absorbância para a temperatura de $20^{\circ} \mathrm{C}$, variando o tempo de agitação e mantendo o tempo de repouso de $6 \mathrm{~h}$.

Tabela 3 - Comparação dos tempos de agitação, $2 \mathrm{~h}$ e $24 \mathrm{~h}$

\begin{tabular}{ccccc}
\hline$t_{a}(\mathrm{~h})$ & $t_{r}(\mathrm{~h})$ & $T\left({ }^{\circ} \mathrm{C}\right)$ & $C_{\text {etanol }}(\%)$ & $A(\mathrm{AU})$ \\
\hline 2 & 6 & 20 & 25 & 0,192 \\
24 & 6 & 20 & 25 & 0,269 \\
\hline
\end{tabular}


sendo que: $t_{a}$ representa o tempo de agitação, $t_{r}$ o tempo de repouso, $T$ a temperatura, $C$ a concentração e $A$ absorbância.

Analisando a Tabela 3, pode-se concluir que o tempo de agitação maior favorece a solubilidade da vitamina $\mathrm{E}$, pois os dados de absorbância foram maiores e, consequentemente, maior a solubilidade.

\subsection{Experimentos com $25 \%$ de etanol}

A construção da curva de calibração para a solução contendo $25 \%$ etanol somente foi possível a partir dos dados coletados através de diversas diluições como pode ser visto na Tabela 4.

Tabela 4 - Dados de solubilidade (S) e absorbância, solução 25\% etanol

\begin{tabular}{ccc}
\hline$\left.C_{\text {solução concentrada }} \%\right)$ & $S \times 10^{6}(\mathrm{~g}$ vit/g solução) & $A(\mathrm{AU})$ \\
\hline 0,5 & 2,480 & 0,068 \\
1 & 5,018 & 0,107 \\
2,5 & 12,54 & 0,175 \\
5 & 24,92 & 0,392 \\
7,5 & 37,55 & 0,562 \\
10 & 50,10 & 0,848 \\
11,5 & 57,30 & 0,961 \\
\hline
\end{tabular}

Com os resultados apresentados na Tabela 4, obteve-se a calibração do sistema, conforme Equação (1), em que o coeficiente de correlação quadrático foi igual a 0,9915.

$$
S=2,1660 \times 10^{-7}+6,0519 \times 10^{-5} \mathrm{~A}
$$

sendo: $S$ a solubilidade da vitamina E (g vitamina/g solução) e $A$ a absorbância (AU).

Foram realizados experimentos para as temperaturas de $20,33,36,7$ e $40^{\circ} \mathrm{C}$. Para cada temperatura estudada colocou-se um excesso de vitamina na célula de equilíbrio juntamente com a solução etanol+água, esta mistura foi deixada em agitação por $24 \mathrm{~h}$ seguidas de $6 \mathrm{~h}$ de repouso. Atingido este tempo, foram coletadas três amostras e medidas as respectivas absorbâncias. Os resultados obtidos estão descritos na Tabela 5.

Tabela 5 - Solubilidade em função da temperatura, solução contendo $25 \%$ de etanol

\begin{tabular}{ccccc}
\hline$T \pm \delta\left({ }^{\circ} \mathrm{C}\right)$ & $A \pm \sigma$ & $\begin{array}{c}A \pm \sigma \\
\text { (após diluição) }\end{array}$ & $\begin{array}{c}S \times 10^{5} \\
\text { (g vit/g solução) }\end{array}$ & Fator de diluição \\
\hline $20,0 \pm 0,2$ & $1,867 \pm 0,031$ & $0,076 \pm 0,011$ & $4,698 \pm 0,600$ & $1: 10$ \\
$33,0 \pm 0,1$ & $0,358 \pm 0,044$ & - & $2,796 \pm 0,200$ & - \\
$36,7 \pm 0,1$ & $1,561 \pm 0,043$ & $0,182 \pm 0,010$ & $11,598 \pm 0,487$ & $1: 10$ \\
$40,0 \pm 0,1$ & $1,273 \pm 0,022$ & $0,116 \pm 0,010$ & $7,313 \pm 0,500$ & $1: 10$ \\
\hline
\end{tabular}

\subsection{Experimentos com $50 \%$ de etanol}

Para a curva de calibração do sistema contendo 50\% de etanol, os dados de solubilidade em função da absorbância $(A)$ estão descritos da Tabela 6. 
Tabela 6 - Dados de solubilidade e absorbância, solução $50 \%$ etanol

\begin{tabular}{ccc}
\hline$C_{\text {solução concentrada }}(\%)$ & $S \times 10^{6}(\mathrm{~g}$ vit/g solução) & $A(\mathrm{AU})$ \\
\hline 1 & 6,819 & 0,039 \\
1,5 & 10 & 0,073 \\
7,5 & 52 & 0,376 \\
10 & 68 & 0,424 \\
12,5 & 85 & 0,525 \\
15 & 102 & 0,601 \\
17,5 & 119 & 0,739 \\
20 & 136 & 0,819 \\
21,5 & 146 & 0,897 \\
22 & 150 & 0,926 \\
\hline
\end{tabular}

Os resultados apresentados na Tabela 6 foram correlacionados, conforme a Equação (2), em que o coeficiente de correlação quadrático foi de 0,9958 .

$$
S=-2,3707 \times 10^{-6}+0,0002 A
$$

sendo: $S$ a solubilidade da vitamina E (g vitamina/g solução) e $A$ a absorbância (AU).

Os experimentos com solução de $50 \%$ de etanol foram realizados de maneira análoga aos experimentos de $25 \%$. Os resultados obtidos estão descritos na Tabela 7.

Tabela 7 - Solubilidade em função da temperatura, solução contendo 50\% de etanol

\begin{tabular}{ccccc}
\hline$T \pm \delta\left({ }^{\circ} \mathrm{C}\right)$ & $A \pm \sigma$ & $\begin{array}{c}A \pm \sigma \\
\text { (após diluição) }\end{array}$ & $\begin{array}{c}S \times 10^{4} \\
\text { (g vit/g solução) }\end{array}$ & $\begin{array}{c}\text { Fator de } \\
\text { diluição }\end{array}$ \\
\hline $20,0 \pm 0,1$ & $2,754 \pm 0,012$ & $0,366 \pm 0,031$ & $9,189 \pm 0,621$ & $1: 10$ \\
$33,0 \pm 0,3$ & $3,513 \pm 0,394$ & $0,607 \pm 0,080$ & $13,894 \pm 1,589$ & $1: 10$ \\
$36,7 \pm 0,1$ & $3,506 \pm 0,084$ & $0,513 \pm 0,084$ & $12,561 \pm 1,067$ & $1: 10$ \\
$40,0 \pm 0,2$ & $3,308 \pm 0,006$ & $0,528 \pm 0,002$ & $12,349 \pm 0,043$ & $1: 10$ \\
\hline
\end{tabular}

No trabalho de Oliveira et al. (2013), os experimentos, também, foram realizados nas frações de 25 e $50 \%$ em diversas temperaturas, assim foi possível comparar os dados obtidos neste trabalho com os dados encontrados na literatura, determinando-se então os desvios relativos, conforme a Tabela 8. Os desvios relativos foram calculados através da Equação (3).

Tabela 8 - Dados obtidos de solubilidade e dados da literatura em função da fração de etanol

\begin{tabular}{ccccc}
\hline$T \pm \delta\left({ }^{\circ} \mathrm{C}\right)$ & $w(\%)$ & $\begin{array}{c}S_{\text {exp }} \times 10^{5} \\
\text { (g vit/g solução) }\end{array}$ & $\begin{array}{c}S_{l i t} \times 10^{5} \\
\text { (g vit/g solução) }\end{array}$ & DR $(\%)$ \\
\hline $20,0 \pm 0,2$ & 25 & $4,698 \pm 0,600$ & $4,719 \pm 0,700$ & 0,447 \\
$20,0 \pm 0,1$ & 50 & $91,890 \pm 0,621$ & $92,850 \pm 0,640$ & 1,045 \\
$33,0 \pm 0,1$ & 25 & $2,796 \pm 0,200$ & $2,836 \pm 0,300$ & 1,431 \\
$33,0 \pm 0,3$ & 50 & $138,940 \pm 1,589$ & $139,050 \pm 1,600$ & 0,079 \\
$36,7 \pm 0,1$ & 25 & $11,598 \pm 0,487$ & $11,629 \pm 0,600$ & 0,267 \\
$36,7 \pm 0,1$ & 50 & $125,610 \pm 1,067$ & $125,850 \pm 1,080$ & 0,191 \\
\hline
\end{tabular}

$$
D R=\left|\left[\left(S_{\text {exp }}-S_{\text {lit }}\right) / S_{\text {exp }}\right]\right| \times 100
$$


sendo: $D R$ o desvio relativo (\%), $w$ a fração mássica de etanol $(\%), S_{\exp }$ a solubilidade da vitamina E experimental (g vit/g solução) e $S_{l i t}$ a solubilidade da vitamina E da literatura (g vit/g solução).

\section{CONCLUSÃO}

Com os resultados experimentais de solubilidade obtidos nesse trabalho, constatou-se que a concentração de etanol em água desempenha grande influência sobre a solubilidade da vitamina E. Foi notório que o aumento da concentração de etanol, aumentou consideravelmente a solubilidade da vitamina E, visto que as soluções com $50 \%$ de etanol apresentaram solubilidades maiores que as soluções com $25 \%$ de etanol, em todas as temperaturas avaliadas.

Notou-se ainda um incremento na solubilidade da vitamina E com o aumento da temperatura, entretanto, estudos e experimentos ainda são necessários para descrever este comportamento, pois nos experimentos com a fração de 50\% de etanol, a solubilidade da vitamina $E$ sofreu oscilações com a alteração da temperatura e obteve-se seu maior valor em $33^{\circ} \mathrm{C}$, diferentemente dos experimentos de $25 \%$, que obteve seu maior valor em $36,7^{\circ} \mathrm{C}$, portanto, não se pode afirmar que a solubilidade aumenta com a temperatura. Quando se comparou os dados obtidos nesse trabalho com os dados disponíveis na literatura, observou-se que os desvios relativos obtidos foram pequenos, indicando que os métodos empregados neste trabalho reproduziram dados condizentes e confiáveis. Embora o tempo de repouso, utilizado nesse trabalho, ter sido maior, constatou-se que os dados obtidos foram próximos aos dados encontrados na literatura.

\section{AGRADECIMENTOS}

Os autores agradecem ao $\mathrm{CNPq}$ pela bolsa de Iniciação em Desenvolvimento Tecnológico e Inovação (PIBIT ITCNPQ 2016-ENG003) e à Faculdade de Engenharia Química da Universidade Federal de Uberlândia pela estrutura física disponibilizada para o desenvolvimento desta pesquisa no Laboratório de Cristalização.

\section{REFERÊNCIAS}

DUBBS, M. D.; GUPTA, R. B. Solubility of vitamin E ( $\alpha$-tocopherol) and vitamin K3 (menadione) in ethanol-water mixture. J. Chem. Eng. Data, 43, p. 590-591, 1998.

GUPTA, R. B.; HEIDEMANN, R. A. Solubility models for amino-acids and antibiotics. AIChE J., 36, p. 333-341, 1990.

MALAGONI, R. A. Determinação experimental da solubilidade de orgânicos em água. 2006. 102 f. Dissertação de Mestrado, Faculdade de Engenharia Química, Universidade Federal de Uberlândia, Uberlândia, 2006.

OLIVEIRA, F.; FONSECA, L. G.; SILVA, C. V.; PIRES, R. F.; MALAGONI, R. A. Solubilidade da vitamina E em mistura etanol+água. In: X COBEQ IC - Congresso Brasileiro de Engenharia Química Iniciação Científica, 2013, Vassouras - RJ. Anais do X COBEQ IC, 2013. p. 1-6.

TRABER, M. G. Utilization of vitamin E. Biofactors, 10 (2-3), p. 115-20, 1999. 